\title{
Strates
}

STRATES Matériaux pour la recherche en sciences sociales

9 | 1997

Crises et mutations des territoires

Table ronde

Quelles dynamiques de l'emploi en milieu rural :
peut-on oser l'expression de «vitalité cachée »?

Nicole Mathieu, Nicole Eizner, Pierre Lenormand, Jeanine Cohen, MarieFrance Épagneul et Jacques Perret

(2) OpenEdition

Journals

Édition électronique

URL : http://journals.openedition.org/strates/638

DOI : $10.4000 /$ strates. 638

ISSN : $1777-5442$

Éditeur

Laboratoire Ladyss

Édition imprimée

Date de publication : 30 septembre 1997

ISSN : 0768-8067

Référence électronique

Nicole Mathieu, Nicole Eizner, Pierre Lenormand, Jeanine Cohen, Marie-France Épagneul et Jacques

Perret, «Quelles dynamiques de l'emploi en milieu rural : peut-on oser l'expression de «vitalité cachée »?», Strates [En ligne], 9 | 1997, mis en ligne le 19 octobre 2005, consulté le 08 septembre 2020.

URL : http://journals.openedition.org/strates/638; DOI : https://doi.org/10.4000/strates.638

Ce document a été généré automatiquement le 8 septembre 2020

Tous droits réservés 


\title{
Table ronde
}

\section{Quelles dynamiques de l'emploi en milieu rural : peut-on oser l'expression de « vitalité cachée »?}

\author{
Nicole Mathieu, Nicole Eizner, Pierre Lenormand, Jeanine Cohen, Marie- \\ France Épagneul et Jacques Perret
}

Nicole Mathieu. - À l'origine de cette table ronde ${ }^{1}$ se trouve la volonté de rendre publique la controverse apparue au sein même du groupe de chercheurs du GDR AGRAL, qui a opposé les signataires du livre entre eux et a provoqué des critiques de ceux qui, comme Nicole Eizner, l'ont lu. Ce clivage se lit aussi dans les échos que L'emploi rural, une vitalité cachée a obtenus. Un certain nombre de comptes-rendus, en particulier celui du Monde ${ }^{2}$, ne retiennent du livre que son sous-titre, Campagne active, une vitalité cachée, mettent l'accent sur le renversement de regard qu'il propose sur l'évolution de l'emploi en milieu rural, relèvent l'existence de possibilités de création d'emplois, y compris dans l'agriculture, à condition de réaliser la difficile synergie entre les «stratégies individuelles, familiales et collectives». D'autres, à l'inverse, reconnaissant une volonté de repérer, au niveau le plus fin, les dynamiques territoriales d'emploi, sont déroutés par la pluralité des approches et des hypothèses et mettent en doute la démonstration prospective et la réalité des créations d'emplois en milieu rural. Il est vrai que le livre s'est construit sur une certaine divergence des hypothèses et des postures théoriques entre les auteurs, préférence pour la réflexion sur les tendances globales pour certains, intérêt pour les faits minoritaires pour d'autres, ce que le titre ne reflète pas. En mettant en avant le terme de vitalité cachée ne risque-t-on pas d'occulter la régression de l'emploi et son corollaire, une régression sociale?

2 Permettez-moi de rappeler avant le début de notre discussion nos points de convergence. Tout d'abord, cette idée : si l'on veut aller plus loin que le discours social et l'analyse scientifique dans la compréhension de l'évolution des rapports entre emploi/chômage et mobilité, ce jusqu'à repérer les incidences concrètes que les 
métamorphoses de la question sociale (Castel, 1995) et la remise en cause des modèles de travail induisent sur les réalités sociologiques d'aujourd'hui, sur les réactions des individus et des groupes sociaux, il faut opérer un renversement de méthode et intégrer dans l'analyse une dimension sous-estimée du problème, la place du territoire et l'analyse géographique. C'est que, de ce point de vue, les dynamiques de l'emploi sont doubles : à la fois ubiquistes et homogènes - de manière équivalente dans tous les pays industriels s'observent la montée du chômage, la restructuration par destruction du travail, la remise en cause du modèle du travail salarié - à la fois diverses et relevant de caractères territoriaux - la délocalisation des entreprises, la décentralisation, sont des processus qui font intervenir la qualité de l'espace, les variations dans le processus d'ensemble sont interprétables par le caractère local du système d'emploi ou par son ancrage territorial. Il nous parait donc important de réexaminer le rapport entre dynamiques de l'emploi et catégorie spatiale, de territorialiser leur analyse.

3 D'où l'intérêt d'analyser l'emploi rural, les dynamiques d'emploi à l'œuvre dans l'espace rural et les types d'espaces ruraux. L'objectif commun s'est alors concentré sur les moyens de surmonter les difficultés de l'analyse de l'emploi en milieu rural dans la mesure où celle-ci se heurte au problème de la définition de la catégorie de «rural » et à l'ambiguïté des représentations qu'on en a. D'un côté, lorsqu'on le considère en termes productifs et comme un espace de travail, l'espace rural apparaît comme le lieu exclusif de la production agricole et l'évolution de l'emploi est pensée au travers de l'agriculture. Ce qui revient à mettre l'accent sur une évolution négative : l'espace rural ne peut être qu'un espace de régression de l'emploi. De l'autre côté, quand on pense l'espace rural du point de vue résidentiel, il ne peut être qu'un espace de diffusion de la ville: la croissance éventuelle de la population ne peut avoir de rapport avec la création d'emplois. En théorie, il ne peut donc y avoir de dynamique positive de l'emploi, de création d'emplois en milieu rural. Nous nous sommes efforcés de sortir de cette théorisation préconçue pour poser frontalement une question qui rassemble l'ensemble des auteurs: quelles sont les dynamiques de l'emploi en milieu rural, positives et négatives, et comment les qualifier?

4 Nicole Eizner. - Ce que je tiens à dire ne s'inscrit pas dans la problématique qui vient d'être dessinée.

5 Votre analyse de l'emploi en milieu rural me semble terriblement technocratique. Il s'agit là d'une réponse à une demande de la DATAR, dénuée de toute perspective critique. Par ailleurs, j'ai eu l'impression que peu importait ce que signifiait la précarité - on parle beaucoup dans ce livre d'emploi précaire et de créativité -, que le niveau des salaires comptait peu, qu'aux abois comme tout le monde on se lançait dans une course à l'emploi sans s'interroger sur le sens de cet emploi, sur ce qu'il signifie dans la vie de quelqu'un et finalement - excusez-moi d'en venir à des considérations banales - sans se poser la question de la rémunération. En d'autres termes, tout ceci me semble aller de pair avec des idées extrêmement à la mode, comme la flexibilité, la mobilité, comme cette précarité généralisée, qui, de plus, ne concernent pas uniquement le milieu rural. De telles considérations m'amèneraient, quant à moi, à des questions comme celle du partage du travail, du problème de l'emploi pour tous, mais surtout pas à m'interroger sur ces «jobs» qui permettent de survivre, quand bien même aurait-on l'avantage de vivre sur son cher territoire et d'habiter, avec toute sa famille, dans ce si charmant petit village... 
6 Autant j'adhère à toutes les idées qui remettent en question le salariat dans sa forme traditionnelle, ou le travail comme moteur essentiel de la vie, autant je ne suis pas du tout prête à penser que tout « job » est une solution, a fortiori quand sont occultées des notions comme l'exploitation du travail, alors qu'il s'agit incontestablement d'un phénomène généralisé. Vous avez tous eu connaissance de ce dernier gadget qui consiste en ce que des entreprises anglaises proposent des travailleurs dans le domaine du tourisme, et notamment des sports d'hiver, à des prix évidemment compétitifs puisqu'ils ne sont soumis qu'aux charges sociales anglaises... Je prétends que ceci est fort dangereux. Si on ne se pose pas le problème de la qualité du travail, du sens qu'il a dans un projet de vie - et non dans un sauve-qui-peut à la recherche d'un emploi - on ne met pas les problèmes de la société à plat, et ce n'est, en définitive, qu'un rapport technocratique qu'on propose.

7 Ce ne sont donc pas tant les problèmes théoriques de relation au territoire, les approches géographiques ou sociologiques, qui m'ont heurtée dans ce livre, c'est proprement le problème politique, la façon d'aborder la notion d'emploi et les significations qu'on lui accorde. J'en verrai pour preuve un certain nombre des recommandations faites à la DATAR, dont une m'a littéralement ahurie : une demande de détaxation de je ne sais quoi ${ }^{3}$, comme s'il fallait permettre à la ruralité de se recréer en ghetto, par des mesures spécifiques, dont on se demande bien pourquoi elles seraient appliquées à la ruralité seule.

8 Nicole Mathieu. - Je ressens d'autant plus fortement ta critique que ce n'était pas notre point de départ. Nous n'avons pas été sollicités par la DATAR pour cette recherche. C'est nous qui lui avons proposé un projet qui, de par sa problématique et dans ses démarches, était conçu pour échapper à tout technocratisme. La proposition que tu dénonces est de François de Ravignan dont on ne peut pas soupçonner le technocratisme. Ta critique est-elle liée au fait que nous n'aurions pas réfléchi sur cette notion d'emploi?

9 Nicole Eizner. - Ce reproche de technocratisme - mais peut-être le mot est-il trop fort ? - est essentiellement lié au fait que selon moi, ce livre n'est pas critique.

10 Nicole Mathieu. - Il s'agit de deux choses différentes. Que la critique en soit absente ne signifie pas que le livre soit technocratique.

11 Nicole Eizner. - Il utilise le terme d'emploi dans des catégories qui sont celles de la DATAR.

12 Nicole Mathieu. - Mais la DATAR n'utilise pas le terme d'emploi à propos du rural !

13 Nicole Eizner. - Disons le carrément: ce livre me semble répondre, dans l'air du temps, à un problème de l'air du temps, il n'y a pas de point de vue politique dans ce livre.

14 Nicole Mathieu. - On distinguera donc les deux critiques. Mettons de côté notre rapport à la DATAR et répondons sur le reproche d'absence de perspective critique et sur la position d'un certain nombre d'entre nous face aux problèmes de l'emploi, de la précarité, de la flexibilité. Ce qui me paraît différencier notre position de la tienne, c'est que, bien que toujours attachés à la théorie marxiste de l'exploitation et de la paupérisation - et à son application aux problématiques de l'emploi - nous sommes un certain nombre qui observons (je reprends à dessein une terminologie de l'observation que tu me reproches souvent) la façon dont les individus, les groupes, tentent de réagir face à ce qui est vécu comme une tendance oppressive : le fait de ne 
pas avoir accès au travail, d'être licencié ou mis au chômage, ou de ne trouver que des emplois précaires ou intérimaires. Car personne, aujourd'hui encore, ne peut la considérer comme quelque chose de positif. Ceci transparaissait dans tous les entretiens que nous avons menés...

15 Nicole Eizner. - Cela va de soi !

16 Nicole Mathieu. - On ne trouve personne qui considère que ce mouvement général, qui a d'ailleurs changé de forme comme le soulignait François Partant, il y a déjà plus de vingt ans, en parlant de destruction du travail, d'une crise...

17 Nicole Eizner. - On sait cela aussi...

18 Nicole Mathieu. - Je le dis malgré tout. Nous avons tenté de rendre compte d'un phénomène individuel de réaction dont l'une des caractéristiques est de repenser le rapport au travail et ce, dans une certaine autonomie.

19 L'oppression et l'exploitation ne sont supportées que jusqu'à une certaine limite, précisément celle de la survie. Lorsque le seuil critique est atteint, lorsque se pose la question de sa survie ou de celle de sa famille, c'est le sens même du travail qui est remis en cause. Mais cette remise en cause n'est pas globale, elle n'intervient que dans la mesure où elle fait partie d'une expérience, d'un vécu.

20 Deux phénomènes très importants apparaissent en milieu rural, et peut-être ont-ils leur équivalent dans d'autres milieux. Hugues Lamarche et François de Ravignan ont mis en évidence le premier qui peut être observé au niveau de l'exploitation agricole. Une tendance s'y manifeste, celle de redonner à cette unité de base une dimension qui n'est pas uniquement celle de la production de revenu, de salaire, mais dont la fonction est de permettre à l'ensemble des enfants de retrouver un sens à leur vie. Ce premier exemple concerne effectivement des gens - ancrés au pays, comme tu le dis - qui concentrent toute leur énergie et intelligence vers un seul but, rendre soluble une question devenue insoluble: celle du devenir de leurs enfants dans le travail. Sur la base de certains segments du procès de travail - transformation de produits, vente à la ferme, hébergement et entretien alimentaire de personnes étrangères à la famille...-, sont ainsi récupérées d'anciennes et de nouvelles ressources et réintégrées dans le procès de travail un certain nombre de tâches qui avaient été complètement expulsées hors de l'exploitation. Tout cet effort, forme post-industrielle de la transmission?, n'est pas fait pour gagner plus d'argent, mais pour que les enfants puissent s'installer, entrer régulièrement dans l'âge adulte.

21 L'autre exemple est inverse car il concerne les gens qui bougent, qui ne sont pas ancrés dans la propriété et le local. Il est intéressant d'observer ces fameux migrants de la décennie 90, ces gens qui quittent la ville et s'installent à la campagne et dont j'ai esquissé les divers profils lors du colloque organisé par José de Félice à Paris 7 en octobre 19964. Il ont certes tous une idée rêvée de la campagne que nature et convivialité idéalise. Mais, à l'instar de la génération post 68 , et qu'ils soient jeunes intellectuels ou sans formation qualifiante, ces migrants ont bien en commun la crise de l'emploi qui suppose, implique, une réflexion sur le travail, sur le sens du travail et de l'exclusion du travail. Une idée fausse de la campagne peut-être, mais qui draine l'intention de ne pas travailler n'importe comment et pour rien; comme s'il valait mieux se mettre à l'écart, contourner, ruser avec la question du chômage, du licenciement, et du travail dépendant. Ce qui apparaît clairement, et dans tous les entretiens, c'est une tentative de repenser le travail dans la relation avec l'ensemble de 
sa vie, le lieu d'habitation, la maison, le paysage et la nature alentour, le voisinage et le réseau familial ou d'affinité. Ce sont certes des points de vue minoritaires, mais qui selon nous sont importants - puisqu'il s'agissait de rendre compte de phénomènes relativement souterrains et que nous n'avions pas l'intention de plaquer un discours extérieur sur la précarité, la flexibilité, mais bien d'essayer, de l'intérieur, d'observer comment les gens se posent le problème de leur emploi et de leur travail.

22 Nicole Eizner. - A cela je ne répondrai qu'une seule chose : un petit nombre de gens se posent la question en ces termes. J'en connais; tu en connais. Ce qui me gêne c'est que leurs propos, et ce qu'ils signifient, soient pris tels quels. Dans une période où de toute part le travail est remis en cause, ces gens s'expriment et parlent comme on s'exprime maintenant. Il y aurait lieu de mener une réflexion sociologique à partir de leur jugement que je ne trouve pas dans ce livre. Peut-être est-ce la raison pour laquelle il m'a semblé technocratique. Par ailleurs, je pense qu'on ne peut traiter ce genre de problèmes sans les replacer dans le contexte économique et social global.

Avez-vous pour hypothèse que ces gens sont porteurs d'un modèle alternatif ? N'est-on pas encore une fois en train de prendre ses désirs pour des réalités? S'agit-il d'autres choses que d'individus? Est-ce un système?

Alors qu'un certain nombre d'exploitations agricoles ajoutent la transformation et la commercialisation à leurs pratiques habituelles, dans le même temps - je me réfère aux articles d'Hélène Delorme et Claude Servolin dans L'Europe et ses campagnes - le GATT et la PAC sont en train de fragiliser non pas les petits exploitants mais la classe moyenne agricole. Il y a toujours partout des mouvements doubles, des phénomènes contradictoires, des poussées dialectiques. J'ai ressenti dans ce livre comme une apologie d'une ruralité innovante, autonome, pourvoyeuse d'initiatives, et ce sans point de vue critique, sans que ce soit pensé au travers d'une dynamique qu'on a de plus en plus de mal à définir et qui toucherait toute la société.

Les gens trouvent assurément les solutions qu'ils peuvent. Mais peut-on en rester là ? S'agit-il de l'amorce d'une nouvelle manière de se situer face au problème du travail ou s'agit-il de minorités ? Il y a déjà quinze ans que des gens retournent en milieu rural pour vivre autrement, le phénomène n'est pas nouveau, même s'il s'est légèrement étendu.

Jacques Perret. - J'aimerais répondre sur l'absence de point de vue politique critique, et sur la question de la précarité et de la flexibilité.

Il est vrai que nous sommes emportés dans un mouvement général de précarité et que chacun fait ce qu'il peut pour s'en préserver. Il y a effectivement une apologie du petit entrepreneur. Parce qu'on a condamné un certain type de modèle, parce qu'on recherche de nouveaux modèles de développement. Parce que nous évoluons en plein brouillard dans le système libéral.

Pourtant ce livre me semble apporter quelque chose sur le plan politique. Il est important, dans la situation actuelle, de mettre en évidence le fait qu'il existe toujours des marges de manœuvre possibles (comme ce fut sans doute le cas dans les périodes de crise antérieures). Sur quoi débouchent-elles? Je l'ignore. Il faudrait se lancer dans la prospective. Cependant on a pu constater par le passé que les marges de manœuvre débouchaient souvent sur des mutations intéressantes. économique dont les développements sont inéluctables, à tel point que le chômage et la 
concentration des activités sont perçus comme des phénomènes irréversibles, il me semble important, sur le plan politique, de constater que le système n'est pas forcément inéluctable, que la concentration des activités n'est pas inévitable, et qu'il ne s'agit pas seulement d'une situation de résistance face à cette machine, pas plus qu'il n'y a lieu d'en attendre l'émergence d'un système alternatif : il y a des forces en œuvre, et elles participent probablement à une recomposition tant spatiale que sociale.

Les acteurs de la recomposition sont partout, à tous les niveaux, et pas seulement dans les sphères de décision ou parmi les entrepreneurs. Mettre cela en évidence me paraît loin d'être, sur un plan politique, anodin; même si par ailleurs ce mouvement ne représente qu'une partie des tendances lourdes qui s'expriment.

31 Nicole Eizner. - Néanmoins je pense qu'il faudrait que nous arrivions, à nous tous, à tenir les deux bouts des phénomènes. Ce que tu dis existe, mais simultanément et également, son contraire. C'est cela une société, un ensemble de phénomènes contradictoires!

Jacques Perret. - Pendant longtemps on a considéré le fait d'être paysan ouvrier comme une situation de survie et de contrainte. Cette situation de misère a existé et perdure sans doute sous d'autres formes. Cependant avec le temps, avec un autre regard, on constate que ces gens qui sont à la fois paysan et ouvrier sont souvent heureux. Peut-être est-on piégé par leur discours, mais pas moins qu'à l'époque où ils se disaient malheureux et prétendaient subir la situation.

Dans une situation de contrainte mais où existent également des opportunités d'emploi et de revenu, je constate que des gens, à leur échelle, ont trouvé des solutions, peutêtre difficiles mais également heureuses. La marge de manœuvre, c'est cette capacité de choix qu'un individu, qu'il soit paysan ouvrier chercheur ou étudiant, développe face à une situation.

34 Je ne suis pas seulement dans une situation de contrainte, je ne suis pas seulement soumis à des pressions économiques, je suis également dans un champ d'opportunités.

35 Pierre Lenormand. - J'ai été d'autant plus attentif aux propos de Nicole Eizner que je me suis toujours senti en porte à faux dans ce groupe de travail. En revanche, j'ai joué le jeu et je continue de le faire, parce que s'y manifestait une diversité d'approches, et qu'il s'agissait d'un lieu où il était possible de s'exprimer librement. J'ai ce travers d'essayer de répondre aux questions qui me sont posées. Ce qui est quelquefois un tort. Il faut aussi savoir répondre que cette question n'est pas la bonne.

Le constat, à la parution de cet ouvrage, de la disparition - qui serait le fait de l'éditeur - du point d'interrogation prévu pour le sous-titre Une vitalité cachée ? m'a rendu furieux. L'interrogation effacée, ce titre témoigne d'une naïveté déconcertante. Il me semble au demeurant que nous avons donné des armes pour que l'éditeur se permette de la supprimer...

Laisser croire en effet (comme Nicole Mathieu l'écrit à la page 153 de la conclusion) à une poussée de l'emploi rural, laisser entendre qu'il y aurait une dynamique positive de l'emploi - et je parle bien de l'emploi rural, c'est-à-dire de la Population Active au Lieu de Travail (PALT) et non des activités des gens qui habitent en milieu rural - est non seulement grave, mais tend à mystifier le lecteur. Il y a des erreurs dans les données. Je rappelle que suivant les chiffres de Jean-Claude Bontron, pour la France rurale, entre 1982 et 1990, la perte nette a été de 360000 emplois, essentiellement dans l'agriculture : -450000 emplois en 8 ans. C'est une catastrophe ! C'est pire que la sidérurgie. 
38 On peut toujours discuter : il y a effectivement un progrès du tertiaire, un progrès relatif de l'emploi secondaire, qui diminue cependant en chiffres absolus dans les campagnes. Ce sont les données extraites de la communication de Jean-Claude Bontron à la SFER en 1994, et reprises (p.52) dans ma contribution à l'ouvrage. Elles n'ont encore été contestées par personne, et je les considère, jusqu'à preuve du contraire, comme une approche correcte de l'évolution récente de l'emploi rural.

En outre, certaines expressions contribuent à entretenir des illusions; nous tombons dans des travers, qui sont effectivement dans l'air du temps, notamment sur le mythe de «l'emploi créé par soi-même ». Ainsi la petite merveille qu'est le titre à la une du Mensuel Challenges ( $n^{\circ} 101$, mars 1996) : «Sortez de la crise, devenez patron. Créer son entreprise, une vraie solution pour l'emploi ». Or, nous savons parfaitement que pour créer du travail il faut non seulement des idées mais finaliser l'emploi - car on le dit maintenant: "solvabiliser l'emploi»- en bref il faut trouver quelqu'un pour le rétribuer. Aussi ces fameux emplois verts demeureront-ils théoriques tant qu'il n'y aura personne pour les rémunérer. Ne nous racontons pas d'histoires!

Si l'on en croit Jacques Perret, ces transformations correspondent à une recomposition, due aux contre-tendances et aux possibilités créatrices de gens soumis à une certaine pression et capables de réagir. Il y a bien entendu du vrai dans cette assertion. Mais rien n'est simple. N'est-ce pas tout autant le résultat de l'application d'une logique du mode de production au stade où il en est, celui d'une recomposition délibérée du travail, et ce, à la campagne comme à la ville ? Dans un numéro de Challenges on peut lire un article de Perdriel parfaitement clair à ce sujet. Il explique que les grands groupes, se recentrant sur leur métier, se sont organisés pour distribuer et diffuser le petit emploi: une sorte de nipponisation du vieux système productif français qui évoque l'essaimage. Cela marche trop bien pour que l'on oublie de dire qu'il s'agit là également d'une stratégie pour ceux qui détiennent les principaux leviers de commande économiques et politiques, puisque, par le biais du capital financier, les contrôles sont extrêmement étroits.

41 Certes, et de tout temps, il y a toujours eu des marges de manœuvre, y compris dans les régimes les plus totalitaires. Il y a aussi, toujours, des opportunités. Mais que nous terminions un rapport à la DATAR par cette recommandation: "Saisissons les opportunités » ne peut que m'inquiéter. Toute ma culture géographique m'entraînerait plutôt vers l'idée d'auto-développement, d'écodéveloppement, de mouvement solidaire. Il est vrai qu'elles ne sont plus à la mode. Elles sont cependant, plus que jamais, vraies et indispensables. Parce qu'il faut prendre la mesure de ce qui se passe actuellement! Et la décomposition, la recomposition, la restructuration autour de nouveaux modes d'organisation du travail, est une donnée non seulement indiscutable, mais planétaire. Si nos réactions ne sont pas à la hauteur de ce qui me semble être une stratégie globale, si nous n'apportons pas des éléments pour y répondre, nous allons tout droit au fiasco.

42 Je ne me satisfais pas de la pluriactivité, quant aux marges de manœuvre, elles ne me suffisent pas.

43 Jacques Perret. - A ceci près qu'il est contradictoire d'affirmer l'existence d'une stratégie globale, pilotée par Perdriel et consorts, et la présence de marges de manœuvre. Prétendre qu'il existe une stratégie globale, c'est se placer dans un mode d'interprétation qui suppose une organisation pilotée. Que faire dès lors? Se mettre de côté ? Éventuellement résister? 

est essentielle. Les élus n'ont que ce mot à la bouche! Nous n'avons jamais connu, de manière aussi durable et approfondie, une telle crise. Et que fait-on ? On bricole. On fait du traitement, parfois économique, mais surtout social. Je me souviens d'un stage à Rambouillet ${ }^{5}$ où nous avions rencontré des gens au prise avec ce type de problèmes à qui nous n'avons pas répondu parce que nous n'avions pas osé parler politique. Nous avons eu tort.

57 C'est ce qui m'intéresse. Considérons ce phénomène ! Les pertes d'avant le recensement de 1970 ne présentaient pas la même résonance puisqu'on observait des transferts d'emploi importants vers d'autres secteurs. Là, le phénomène est général, hormis cette poussée du tertiaire pour l'emploi rural. 
58 Nicole Mathieu. - De quoi dispose-t-on pour clarifier la question des dynamiques de l'emploi en milieu rural? Selon les sources les données sont différentes, voire contradictoires. Les chiffres avancés par Jeanine Cohen émanent de l'Enquête Structures des Emplois. Bien qu'ils soient limités aux établissements de plus de 20 salariés et excluent les emplois de l'Administration, ils montrent une progression des emplois des entreprises entre 1985 et 1988 pour le seul niveau des communes rurales $(+12000)$ alors que toutes les unités urbaines voient leurs effectifs diminuer sévèrement. Les chiffres avancés par Jean-Claude Bontron et la SEGESA s'appuient sur les Recensements de Population et font état d'une diminution globale de 388500 emplois entre 1982 et 1990. Or ce document ne précise pas sur quelle enveloppe spatiale ces chiffres sont élaborés. S'agit-il de l'enveloppe 1990 des communes rurales ou de celle de 1982 ? En effet, sur la même base des recensements, les données avancées par l'équipe INRA de Dijon sont différentes. C'est la troisième source, sur laquelle je me suis appuyée, Actifs employés au lieu de travail, 1982-1990, délimitation INSEE $1982{ }^{6}$. Elle donne une progression des emplois ruraux dans les communes rurales in ZPIU (+63 000) et une diminution dans le rural hors ZPIU (-223 000), ce qui aboutit à un solde négatif, certes, mais cependant inférieur au chiffre avancé par Jean-Claude Bontron. Toutes les sources mettent en évidence la progression des emplois non agricoles et tous les auteurs signalent la progression à contre tendance des emplois industriels en milieu rural.

Ce que je retiens de votre critique : au lieu de mettre l'accent dans ma conclusion En guise de synthèse sur le fait que l'on repérait des dynamiques positives, il eut été plus rigoureux de préciser les choses telles qu'elles sont : les chiffres sont contradictoires, et il existe une controverse - certains, stigmatisant les pertes catastrophiques d'emploi en milieu rural, minimisent la dynamique même de l'emploi alors que d'autres la soulignent - une controverse liée aux idéologies sous-jacentes à l'interprétation des chiffres. Il me semble important, personnellement, de montrer que dans cet espace les tendances globales à la destruction du travail et à la diminution des emplois industriels sont contrecarrées.

On échappe difficilement à la nécessité de prendre parti et au désir de contredire ce discours dominant selon lequel, en milieu rural, il y a non seulement une diminution des emplois mais que cette tendance ne peut que s'aggraver.

61 Nicole Eizner. - Il est curieux que tu prononces le terme de discours dominant, alors que ce livre se situe, à un autre niveau, pleinement dans le discours dominant.

62 Nicole Mathieu. - C'est précisément ce que je m'efforce d'expliquer. Nous sommes dans une position contradictoire, étant à la fois critique à l'égard du discours dominant et finalement...

Jeanine Cohen. - Alors que finalement nous y revenons lorsque nous nous faisons l'écho du «Sortez de la crise, devenez patron ».

Cf. Doc annexe : L'évolution de l'emploi en milieu rural.

Nicole Mathieu. - Le discours dominant, c'est que l'emploi en milieu rural est condamné!

67 Pierre Lenormand. - Mais la situation est identique, en ville comme à la campagne !

68 Nicole Mathieu. - Non! pas au plan idéologique. Ce que je vous reproche, c'est de constamment changer de niveau, d'amalgamer l'idéologie et l'analyse des faits. 
69 Pierre Lenormand. - Il serait cependant difficile d'oublier que la campagne fait partie de la société globale.

70 Nicole Mathieu. - Ce n'est pas pour autant qu'il faut s'abstenir de l'analyse de ce qui s'y passe. Et c'est ce que nous tentons de faire avec obstination.

71 Jeanine Cohen. - En ce qui me concerne, bien que coauteur du livre, je ne suis pas ruraliste et je ne me suis pas du tout référée aux polémiques en cours dans les milieux spécialisés. En outre, j'ai toujours essayé d'articuler mon engagement politique avec une volonté de ne pas tomber dans le sectarisme et de ne pas opérer de confusion entre le militantisme, fondé sur des choix idéologiques, et la vérité scientifique telle qu'elle se dégage d'observations nouvelles. Je considère qu'il faut s'astreindre à regarder scrupuleusement les chiffres et à dire ce qu'on y voit sans extrapoler, pour ne pas risquer, par d'éventuelles déductions trop hâtives, de dévoyer le résultat et les conclusions que l'on peut tirer de ce qu'on a réellement vu. Tout doit débuter par un effort de description. J'ai donc toujours axé mon travail professionnel sur une volonté de considérer les chiffres, aussi humblement que possible, et de faire part de mes constatations. Peut-être ne correspondent-elles pas à ce que j'attendais, je ne suis peutêtre pas à même de les interpréter ? Ces observations, pourtant, sont ce que je vois. Ce qui permet, avantage non négligeable, d'accéder à la complexité du réel.

72 Il fallait, d'une part, considérer le phénomène spatial majoritaire, qui, dans cette phase de mondialisation de l'économie, tourne autour des questions de polarisation. En effet, la relance de la division du travail à l'échelle mondiale, actuellement maximale, pousse à des polarisations très fortes. De ce fait, se donner le milieu rural comme terrain d'observation, c'est s'astreindre à considérer des espaces fortement soumis à des pressions et incitations qui leur sont extérieures. Et d'autre part il fallait tenir compte de ce que, dans l'espace rural proprement dit, le phénomène majeur est effectivement cette chute catastrophique de l'emploi agricole traditionnel. Ces perspectives peuvent toutes deux conduire à une acceptation de l'idéologie dominante : le discours pour la première est alors: "Sortez de la crise, devenez patron " et ce, appliqué à des populations situées dans une orbite urbaine (la très grande majorité de la population est dans cette situation-là, y compris les habitants de certaines communes rurales); et pour la seconde le discours est du type : "Sans doute êtes-vous malmenés et mis en difficulté, mais au moins vous avez ce petit coin de nature, une certaine marge de manœuvre, richesses qui, pour le moment, ne vous sont pas ôtées ». Ce sont deux discours dominants, qu'il faut bien entendu critiquer car ils sont loin d'être les conclusions obligées et valables des nouveaux problèmes mis au jour. Si nous avons commis une erreur, c'est peut-être celle de ne pas avoir fermement exprimé de quel point de vue nous partions. D’un point de vue critique, évidemment ! Car les efforts d'adaptation sont contrariés par le recul général de l'emploi. Mais pourtant ces efforts d'adaptation ne se soldent pas que par un bilan d'échec. Ils sont également porteurs d'innovation et d'avenir. Avons-nous, alors, en le disant, versé dans le discours dominant ? Je ne le crois pas. Il me semble que sur la catastrophe de l'emploi agricole le texte de Hugues Lamarche et de Marie-France Épagneul est clair. Je crois avoir été assez claire également sur le fait que les dynamiques dominantes accentuent l'inégal développement de la campagne et de la ville.

73 Les chiffres ne sont contradictoires qu'en apparence; plutôt que de se livrer, dans une controverse, à de douteuses manipulations au nom des différentes idées que l'on défend, livrons les deux aspects des choses. D'une part ce qu'ont dit Jean-Claude 
Bontron et Sylvie Cabanis est tout à fait juste. Il est vrai que l'espace rural proprement dit, selon les critères très précis de l'INSEE, a perdu un nombre considérable d'emplois, il est vrai qu'il s'agit là d'une catastrophe qu'il n'est pas question de garder sous silence. Il est aussi vrai que l'enveloppe de cet espace rural a considérablement diminué entre 1975 et 1982, ce qui a pour conséquence d'amplifier le mouvement de perte. Cette combinaison de modifications fait qu'il n'est pas possible de cerner suffisamment finement le bilan exact des pertes d'emploi de la société rurale. En revanche, si l'on confronte, à « enveloppe spatiale » identique, l'espace rural de 1975 à celui de 1982, on constate qu'il montre un bilan positif d'emplois. Pourquoi ? Parce qu'une bonne partie de ses communes, tout en restant rurales, sont nouvellement tombées dans la dépendance d'une zone urbaine et se sont développées: une augmentation principalement générée par la croissance urbaine. Les chiffres ne sont contradictoires qu'en ce sens qu'ils révèlent deux tendances qui se contrecarrent l'une l'autre. Mais l'espace rural ne peut se défendre contre l'expansion urbaine, c'est un espace qui, dernièrement, sert à installer de nouveaux emplois alors que les centres villes sont en perte de vitesse. Nous sortons de décennies où les gens ont, comme on dit, «brûlé leur chandelle par les deux bouts", se livrant à une exploitation bien trop exagérée de toutes les ressources existantes sans considération des questions de reproduction et de legs à la nouvelle génération.

Tout ce que j'ai pu lire dans les comptes-rendus de presse à propos du livre me semble traduire ce besoin qu'ont de nombreuses personnes de s'accrocher à une alternative possible et au besoin d'espérer. Je ne serais pas aussi critique que Nicole Eizner à l'égard de Jacques Perret; s'il existe une marge de manœuvre dans la tourmente que nous vivons, si infime soit-elle, je préfère regarder cette petite lueur qui se dessine au fond du tunnel, que de la tenir pour un leurre en me cantonnant à dénoncer la crise.

75 Nicole Eizner. - Je suis très mal à l'aise face à cette dichotomie rural-urbain. Tracer le portrait d'une campagne dévorée par la ville m'a toujours semblé bizarre. Qu'il y ait eu un mouvement de l'histoire certes, mais dans la société actuelle cette dichotomie n'explique pas grand chose. Ces fameuses initiatives en milieu rural ne sont la plupart du temps - témoins en sont ces thématiques de l'entreprenariat rural à partir de l'exploitation agricole - que le fait de petits patrons qui diversifient leurs activités. Or il en va de même pour mon boucher ou mon boulanger à Paris, qui deviennent traiteurs, font des sandwichs... C'est intéressant, en tant qu'initiatives de petites classes moyennes face à une crise qui demande de se débrouiller. Et une bonne part de la pluriactivité, c'est cela aussi.

76 En ce moment cependant j'ai besoin, non pas de m'interroger sur les spécificités du milieu rural en matière d'autonomie dans la recherche du travail, mais de voir clair face à cette question : «Où allons-nous globalement? ». C'est en ce sens que je regrette que cet ouvrage n'offre pas de réflexivité critique ; ce qui n'enlève rien à la valeur des initiatives des uns et des autres. Il n'y a pas là de contradiction, et comme l'a dit Pierre Lenormand, c'est même l'un des aspects de la mondialisation.

77 Ce n'est tout de même pas un hasard si le capitalisme - pour employer des grands mots - nous serine depuis dix ans la nécessité des initiatives locales!

78 Pierre Lenormand. - Quand je mets en cause le fait qu'au centre de la réflexion de Jacques Perret il y ait les marges de manœuvre et l'opportunité, ce n'est pas, bien entendu, les gens et leurs initiatives que je vise. Il n'est pas question de critiquer le fait que des gens se débrouillent. Cependant ma responsabilité de chercheur, ma 
responsabilité de citoyen, ne se satisfont ni d'opportunités ni de marges de manœuvre face à cette question : « Où va-t-on? ». Ce n'est pas ainsi que nous modifierons quoi que ce soit. Parce qu'il me semble nécessaire de changer les choses! Il faut que nous prenions un recul indispensable pour proposer une démarche globale.

79 Nicole Eizner. - On peut étudier le milieu rural, au même titre qu'un autre, et en tirer des enseignements globaux...

80 Jacques Perret. - Je suis sûr que si nous mêlions nos analyses, nous pourrions nous entendre. Les tendances lourdes sont plus prégnantes que les marges d'autonomie, cela va de soi. N'importe quel article de ce livre, si on veut bien le lire, le reconnait. Et quand bien même! Tant mieux si ce n'est pas dit, nom d'une pipe! Enfin on pourra espérer quelque chose ! Je ne veux pas mourir désespéré !

81 Par ailleurs je tiens à dire, suite à l'intervention de Jeanine Cohen, que je ne crois pas à l'objectivité concernant les chiffres: nous les abordons tous avec des hypothèses de travail.

82 Jeanine Cohen. - Une précision à l'intention de Nicole Eizner: la plupart de ces contributions ont été écrites en 1991 et reprises en 1993. Depuis les choses sont allées en s'accélérant. On sait que nous sommes dans une phase d'interactivité et de dynamique très forte, comme en témoigne par exemple, l'accélération de l'intégration européenne. Peu d'auteurs tiraient alors la sonnette d'alarme, alors que cela a tout de même été fait dans notre livre, par exemple par François de Ravignan.

83 Nicole Eizner. - Je peux résumer ma critique en deux mots : il manque à cet ouvrage deux ou trois chapitres de réflexion sur le sens de tout ce qui y est dit. Ce livre n'est pas fini.

84 Nicole Mathieu. - Je suis entièrement d'accord. C'est d'ailleurs la raison pour laquelle je tenais absolument à ce que nous fassions cette table ronde: les critiques à son endroit me semblant essentielles pour aller plus loin. Je crois, a posteriori - et je ne pense pas que nous en ayons eu véritablement conscience quand nous avons décidé de le publier - que nous n'avons pas été jusqu'au bout de la prise de conscience du fait que nous menions parallèlement deux types de combats, avec en outre des positions différentes au sein du groupe pour chaque combat. Le premier était scientifique, nous tenions à prendre position sur la controverse concernant l'évolution du milieu rural et de l'emploi rural, et donc à prendre position sur la question de la ruralité. Ce combat-là était lui-même contradictoire puisque certains pensent que la dichotomie rural-urbain n'ayant plus de sens il n'y a pas lieu de la travailler, tandis que d'autres considèrent que la différenciation et la spécificité sont porteuses, dans l'analyse, de quelque chose de positif. On aboutissait donc à deux points de vue différents. Dès l'instant où l'on tient à mettre la polarisation et la diffusion en évidence, la dynamique n'est interprétée qu'en terme de domination urbaine. On peut avoir envie, cependant, de montrer qu'il existe des phénomènes liés aux spécificités de la ruralité, à savoir sa faible population, sa faible densité, la question des paysages et de la nature. D'où les métiers verts, l'environnement, etc.

85 Le second type de combat portait sur la controverse politique et sur la position à adopter face à la question du salariat, à celle de sa disparition ou de sa résistance, et au concept de travail. Et je pense que nous n'avions pas pris toute la mesure de nos divergences à propos de cette seconde controverse. Deux tempéraments se sont manifestés, celui de Pierre Lenormand qui considère comme dangereux d'effacer les 
notions d'exploitation, de domination, de mondialisation parce que, selon lui, ces problèmes sont prioritaires et primordiaux. Et de l'autre côté, le tempérament de Jacques Perret qui se porte vers les « gens de peu » et leur intelligence quand il s'agit de reprendre un minimum d'autonomie face à cette domination.

Ce débat nous a obligé à voir que la position du chercheur, toujours complexe, l'est dans ce cas particulièrement, parce qu'il doit intégrer à la fois le plan du rural et celui de la question du travail en général, et également des différences de conception dans la formulation de ce qu'est l'idéologie dominante. Le chapitre qu'il faudrait écrire devrait précisément traiter de la pluralité de ces approches, et également poser cette question : sommes-nous prêts ou non à accepter le fait que l'observation de ce qui se passe dans la réalité viennent déstabiliser nos positions théoriques ? Il y a là quelque chose qui nous différencie. Est-on capable de rendre compte de phénomènes qui ne s'insèrent pas dans l'idéologie dominante?

87 Nicole Eizner. - C'est le propre de notre métier. Et les uns et les autres nous ne faisons que ça!

\section{EUOLUTION DE L'EMPLOI RURAL ENTRE 1982 ET 1990}

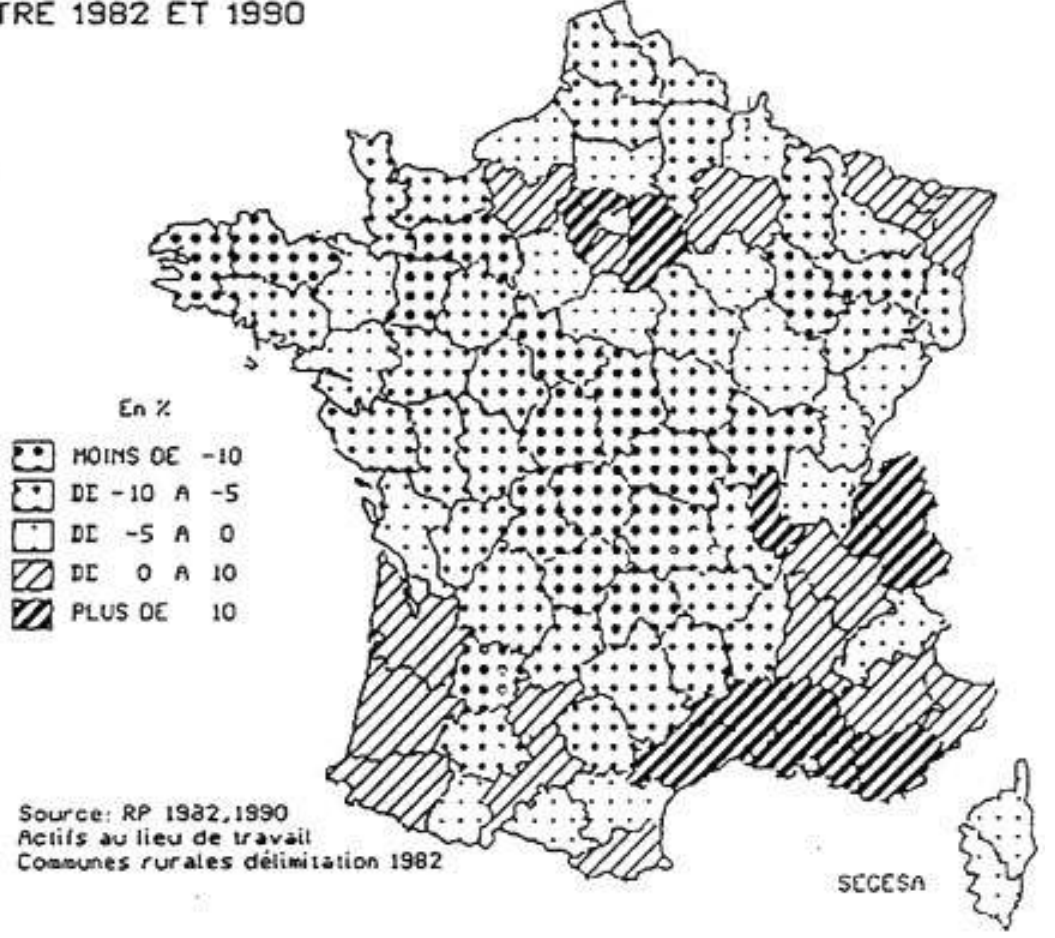

88 Nicole Mathieu. - Qu'il s'agisse de chiffres ou d'informations, nous sélectionnons. Sommes-nous prêts à sélectionner des phénomènes qui nous déstabilisent totalement sur le plan théorique?

89 Pierre Lenormand. - Il ne faut pas raconter d'histoire à propos des sources, nous en avons, nous pouvons les utiliser et réfléchir sereinement. J'ai sous les yeux une carte, incontestable, de l'évolution des "actifs au lieu de travail, communes rurales, délimitation 1982 ». Et elle est fondée sur la même délimitation en 1982 et 1990 . C'est justement les inégalités qui sont marquantes. Hormis une large région parisienne, un nord-est frontalier, les grandes Alpes en général et un petit peu de côte atlantique, c'est la catastrophe.

Nicole Mathieu. - La catastrophe t'intéresse ! 
91 Pierre Lenormand. - Bien sûr ! J'en tiens compte assurément, certains en souffrent, quoique ce ne soit ni arithmétique, ni mécanique, et qu'un emploi perdu ne signifie pas nécessairement un drame. Ce qui ne m'a pas empêché bien sûr de souligner les disparités : elles sont constitutives de notre modèle de développement.

Comment peux-tu nous mettre en garde sur nos capacités à accepter d'être déstabilisé alors que nous ne faisons que cela! Que je le veuille ou non, dès que j'allume la télévision ou la radio, lorsque je discute avec mes collègues, sans cesse et quotidiennement, mes éventuels a priori théoriques sont complètement remis en question. Je ne peux pas faire autrement qu'être tous les jours déstabilisé !

Il y a certes deux niveaux, le scientifique et le politique, mais il me déplaît de porter une casquette dans certains endroits seulement et non dans d'autres. Comment fais-tu pour te couper en morceaux? Les questions scientifiques et politiques sont naturellement liées, et naturellement nous faisons des choix, le plus honnêtement possible bien entendu.

94 Nicole Eizner. - Je n'ai pas besoin d'être déstabilisée : je ne sais plus. Pour ce qu'il en est des positions théoriques...

95 Nicole Mathieu. - C'est précisément ce que j'ai voulu dire.

96 Nicole Eizner. - Cependant, quand j'observe le réel, que ce soit le milieu rural ou l'environnement, je ne me départis pas de la seule question qui m'intéresse : où vont nos sociétés? Le rural en soi, l'environnement en soi, m'importent peu. Il n'est pas possible, particulièrement aujourd'hui, de se targuer de ses données, de se prétendre scientifique pour avoir observé tel ou tel phénomène, si on ne se pose pas la question du sens de tous ces faits dans un mouvement.

97 Nicole Mathieu. - Ce que je proposais, c'était presque une méthode de travail pour arriver à ce que je reconnais moi aussi comme étant l'essentiel, à savoir la leçon que l'on peut en tirer pour l'évolution de nos sociétés. Je crois qu'il est fondamental de commencer par distinguer nos niveaux de travail, d'arriver à exprimer au plus près ce sur quoi nous avons des résultats véritablement exacts, et ce qui fait partie de notre idéologie. Nous devons en être capables. Il n'y a pas parmi nous homogénéité, il est important de le savoir, et c'est positif. Comprenez bien qu'il ne s'agit pas de hiérarchiser ni d'affirmer que certains ont plus raison que d'autres. Mais il me semble que du décodage franc de nos positions dans les différents niveaux peut sortir une vision plus claire de nos réflexions, qui ne se fonde pas sur une déstabilisation et sur une incertitude complète quant à la manière dont on voit l'avenir.

Nicole Eizner. - Pour conclure de façon cavalière, ce livre m'a semblé être une défense et illustration du monde rural, approche qui me semble aussi être parfaitement idéologique. 


\title{
Campagne active
}

\begin{abstract}
L'EMPLOI RURAL - UNE VITALITE CACHEE
sous la direction de Nicole Mathieu.

L'Harmattan, 186 p., 110 F.

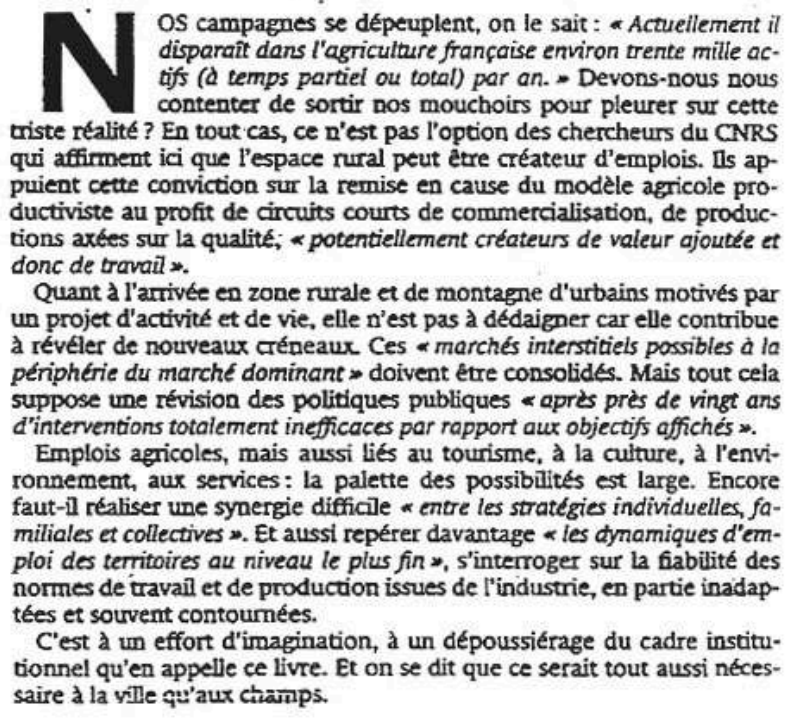

Dariel Urbain

Le Monde du 26/6/1996

\section{NOTES}

1. Table ronde animée par Nicole Mathieu autour de Nicole Eizner en présence des auteurs de L'Emploi rural, une vitalité cachée (Nicole Mathieu dir., 1995, Paris, L'Harmattan) : Jeanine Cohen, Marie-France Épagneul, Pierre Lenormand, Nicole Mathieu, Jacques Perret. Texte établi par Armelle Auris.

2. Le Monde du 26/6/1996 (cf. p. 210).

3. Cf. p. 159 : «C'est pourquoi le groupe a préféré conclure l'exercice prospectif en avançant des propositions de mesures et d'actions : - l'amélioration du dispositif statistique et la mobilisation autonome des données par les chercheurs pour vérifier au niveau national les hypothèses révélées par les analyses locales; - des mesures levant les freins institutionnels à la création d'emploi et à l'installation en milieu rural du type prix de l'essence, des télécommunications (téléphone et fax), allégements fiscaux etc.; l'encouragement par la formation au retour à l'emploi pluriactif."

4. Cf. Habiter à la campagne : mode ou mouvement social ? in Colloque « Nouveaux types de départs de la ville vers la campagne », 18-19 octobre 1996, Université Paris 7 Denis Diderot.

5. Cf. Le stage de formation continue en 1996 à la Bergerie nationale de Rambouillet, «Emploi, nouvelles ressources en milieu rural » : organisé par Nicole Mathieu (STRATES) et Marie-France Epagneul (GRMSE Nanterre) à la demande du ministère de 
l'Agriculture (M. Hayats, DGER) ; pour des directeurs et enseignants de Lycées agricoles, des animateurs de développement rural, des élus ; durée une semaine ; intervenants du groupe AGRAL/DATAR : P. Lenormand, J. Cohen, A. Cazin-Kenny, C. Balley, J.P.

Montalieu, N. Le Coz de STRATES, J. Perret du CEMAGREF Grenoble, De Ravignan INRA Toulouse, Y. Négro de l'UFR Géographie Toulouse.

6. Cavailhès Jean et al., 1994, Analyses des évolutions récentes de l'espace rural, Économie rurale, 223

\section{RÉSUMÉS}

La controverse joue un rôle essentiel dans les résultats et le progrès des sciences sociales. L'Emploi rural, une vitalité cachée, cette recherche collective et pluraliste sur l'avenir des emplois dans l'espace rural français publiée fin 1995, n'a pas épuisé les divergences d'interprétation sur le sujet et continue, une fois achevée, à opposer même les auteurs entre eux. L'objectif de cette table ronde, qui s'est tenue en décembre 1996, fut d'approfondir ces désaccords par leur discussion. Dans ce débat, chacun cherche, dans une langue vive et polémique, à préciser sa pensée en même temps que ce qui fait contradiction. On aboutit pourtant à un consensus : pour avancer plus loin dans l'analyse de phénomènes sociaux encore incertains, il faut théoriser les désaccords et prolonger la recherche à plusieurs voies.

Dynamics of the employment in rural areas : may we speak of a vitality?

This paper relates the debate organized after the publication of L'Emploi rural, une vitalité cachée?, in order to cross the contradictory points of view of the authors in the face of an outward sociologist reader. Though it was quite hot, the discussion has deepened the analysis and opened up to new approaches and questions.

INDEX

Mots-clés : mutations, emploi rural, crises, paupérisation, initiatives locales, acteurs locaux Keywords : rural areas, dynamics of employment, theories, controversy

\section{AUTEURS}

\section{NICOLE MATHIEU}

Nicole MATHIEU est directeur de recherches au CNRS (LADYSS). Avec une spécialisation de géographie rurale et sur les problèmes dits d'environnement, elle s'attache à promouvoir une conception de la recherche donnant une valeur à l'expression de la subjectivité, aux pratiques collectives, à l'interdisciplinarité. Sa démarche privilégie un va et vient entre différents niveaux (analyses locales, analyses générales en France et en Europe). 


\section{NICOLE EIZNER}

Nicole EIZNER est directeur de recherche honoraire au C.N.R.S. Sociologue rurale, elle est spécialiste des représentations liées au milieu et à l'environnement. Elle est présidente de l'Agence nationale de création rurale et du Gerdal (Groupe d'expérimentation et de recherche Développement et Actions localisées).

\section{PIERRE LENORMAND}

Pierre LENORMAND, maître de conférences de géographie à l'Université Paris VII, travaille sur les transformations contemporaines des campagnes méditerranéennes et de leurs marges (Maghreb, Europe du nord-ouest, Europe du sud-est et Balkans).

\section{JEANINE COHEN}

Jeanine COHEN est chargée de recherches au CNRS (LADYSS). Elle étudie les dynamiques spatiales de l'emploi liées au changement dans la géographie du système productif. Ses derniers travaux portent sur les dynamiques des entreprises de la zone centre de la Seine-Saint-Denis et la comparaison des dynamiques technopolitaines dans les régions parisienne et londonienne.

\section{MARIE-FRANCE ÉPAGNEUL}

CNRS

\section{JACQUES PERRET}

Jacques PERRET est chargé de recherche au CEMAGREF de Grenoble. Economiste, il est spécialisé dans les processus de développement touristique. Ses travaux récents ont porté sur le marché du travail et les pratiques d'emploi, notamment la pluriactivité et l'emploi saisonnier, dans leur dynamique territoriale. 